\title{
EPR Study of the Two-Dimensional Quantum System $\mathrm{Cu}(\mathrm{en})\left(\mathrm{H}_{2} \mathrm{O}\right)_{2} \mathrm{SO}_{4}$
}

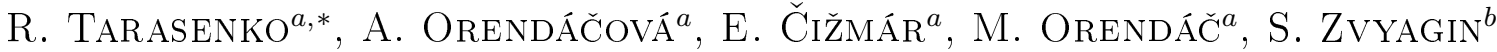 \\ AND J. WOSNITZA ${ }^{b}$ \\ ${ }^{a}$ Centre of Low Temperature Physics of P.J. Šafárik University and SAS \\ Park Angelinum 9, SK-041 54 Košice, Slovak Republic \\ ${ }^{b}$ Dresden High Magnetic Field Laboratory (HLD), Helmholtz-Zentrum Dresden-Rossendorf \\ 01314 Dresden, Germany
}

\begin{abstract}
The angular dependence of electron paramagnetic resonance spectra of $\mathrm{Cu}(\mathrm{en})\left(\mathrm{H}_{2} \mathrm{O}\right)_{2} \mathrm{SO}_{4}$ single crystals was studied in the X-band frequency range at temperatures 4 and $300 \mathrm{~K}$. Analysis of the linewidth at $300 \mathrm{~K}$ revealed nice agreement with the angular variation of the $g$-factor. This coincidence is the manifestation of the symmetric and antisymmetric exchange coupling, as main broadening mechanisms in $\mathrm{Cu}(\mathrm{en})\left(\mathrm{H}_{2} \mathrm{O}\right)_{2} \mathrm{SO}_{4}$ at high temperatures. The radical change of the angular dependence of the linewidth observed at $4 \mathrm{~K}$ can be ascribed to dipolar coupling.
\end{abstract}

PACS: $76.30 . \mathrm{Fc}, 75.40 .-\mathrm{S}$

\section{Introduction}

Two-dimensional (2D) quantum magnets have been extensively investigated due to their unconventional magnetic properties resulting from the interplay between quantum fluctuations and geometrical frustration [1]. Electron paramagnetic resonance (EPR) spectroscopy plays an important role in studies of these systems (see for instance $[2,3])$.

In this paper, we report on the angular dependence of EPR spectra of $\mathrm{Cu}(\mathrm{en})\left(\mathrm{H}_{2} \mathrm{O}\right)_{2} \mathrm{SO}_{4}$ single crystals investigated at two temperatures, 4 and $300 \mathrm{~K}$. The title compound has been previously identified as a potential realization of the quasi-two dimensional spatially anisotropic triangular Heisenberg antiferromagnet with spin $1 / 2$ and effective intralayer exchange coupling, $J / k_{\mathrm{B}} \approx-1.4 \mathrm{~K}$. A phase transition to a magnetically ordered state has been observed in zero magnetic field at $T_{\mathrm{N}}=0.91 \mathrm{~K}[4]$.

\section{Experimental details}

The EPR measurements have been performed at the Dresden High Magnetic Field Laboratory using an X-band spectrometer (Bruker ELEXSYS E500) at a fixed frequency of $9.4 \mathrm{GHz}$ in magnetic fields up to $0.5 \mathrm{~T}$ and at

* corresponding author; e-mail:

robert.tarasenko@student.upjs.sk
4 and $300 \mathrm{~K} . \mathrm{Cu}(\mathrm{en})\left(\mathrm{H}_{2} \mathrm{O}\right)_{2} \mathrm{SO}_{4}$ (en = ethylendiamine $=$ $\mathrm{C}_{2} \mathrm{H}_{8} \mathrm{~N}_{2}$ ) single crystals have been prepared using a procedure described in Ref. [5]. The crystals had typical dimensions $a^{\prime} \times b^{\prime} \times c^{\prime}=4 \times 1.5 \times 0.5 \mathrm{~mm}^{3}$. The crystal structure (Fig. 1) at $300 \mathrm{~K}$ is monoclinic (space group $C 2 / c$ ) with the lattice parameters $a=7.232 \AA$, $b=11.752 \AA, c=9.768 \AA, \beta=105.50^{\circ}, Z=4[5]$.

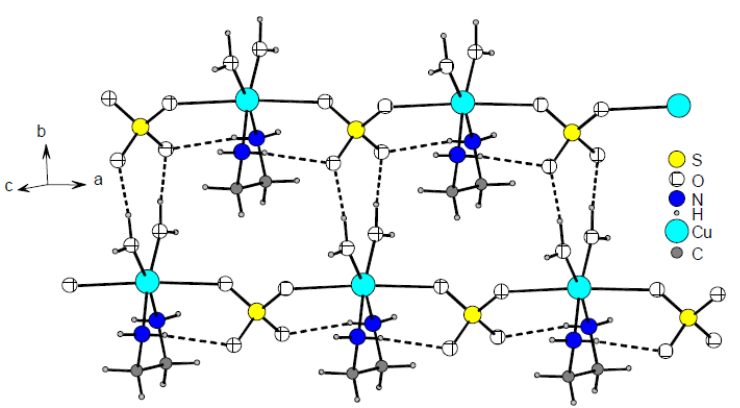

Fig. 1. Crystal structure of $\mathrm{Cu}(\mathrm{en})\left(\mathrm{H}_{2} \mathrm{O}\right)_{2} \mathrm{SO}_{4}$. The dashed lines represent hydrogen bonds.

Preliminary neutron studies [6] identified the $a c$ crystallographic plane with a flat surface $a^{\prime} b^{\prime}$, while the crystallographic axis $b$ coincides with the $c^{\prime}$ edge. The angular dependence of the EPR spectra was investigated by rotating the crystal within the planes $b^{\prime} c^{\prime}, a^{\prime} c^{\prime}$, and $a^{\prime} b^{\prime}$. The shape of the resonance lines was described by a Lorentzian, which was used for a fitting procedure with two fitting parameters, i.e., the resonance magnetic field 
and the linewidth. $g$-factors were calculated from the resonance magnetic fields using the resonance condition $\hbar \omega=g \mu_{\mathrm{B}} B$.

\section{Results and discussion}

The EPR spectrum consists of a single resonance line in all orientations at room temperature as well as at low temperature (Fig. 2). This observation is in agreement with structural studies which indicate that all $\mathrm{Cu}$ (II) ions occupy crystallographically equivalent positions. Thus, the magnetic subsystem of $\mathrm{Cu}(\mathrm{en})\left(\mathrm{H}_{2} \mathrm{O}\right)_{2} \mathrm{SO}_{4}$ can be treated as an assembly of identical ions with the same $g$-factors. The angular dependences of the $g$-factor were fitted using the standard relation $g^{2}=g_{z}^{2} \cos ^{2} \theta+$ $g_{x}^{2} \sin ^{2} \theta \cos ^{2} \varphi+g_{y}^{2} \sin ^{2} \theta \sin ^{2} \varphi$, where $g_{x}, g_{y}, g_{z}$ are $g-$ -factors corresponding to the local anisotropy axes, $\theta$ is the angle between the magnetic field and the local $z$ axis and $\varphi$ is the angle between the projection of the magnetic field in the plane $x y$ and the local $x$ axis. The $\mathrm{Cu}(\mathrm{II})$ ion is octahedrally coordinated, thus the local axes can be identified with the axes of the octahedron. In the first approximation, on the basis of the knowledge of the crystal structure, the local $z$ axis was identified as the $a^{\prime}$ edge and the $x y$ plane as the $b^{\prime} c^{\prime}$ plane. The angles in the fitting relation were corrected by the experimental angle $\alpha$ due to the existence of a tilting angle between the edges of the crystal and local anisotropy axes. The results of the fitting procedure are presented in Table.

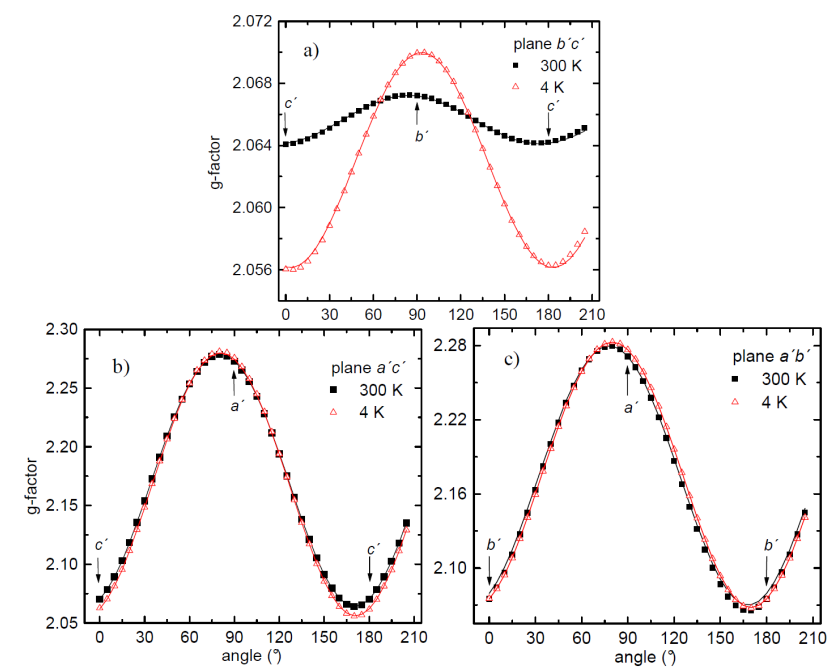

Fig. 2. Angular dependence of the $g$-factor of $\mathrm{Cu}($ en $)\left(\mathrm{H}_{2} \mathrm{O}\right)_{2} \mathrm{SO}_{4}$. The lines represent the fit described in the text.

The analysis of the $g$-factor confirmed the elongation of the local octahedron surrounding the $\mathrm{Cu}(\mathrm{II})$ ion. Furthermore, the enhancement of the difference between the minimal and maximal values of the $g$-factor in the $x y$ $\left(b^{\prime} c^{\prime}\right)$ plane observed at $4 \mathrm{~K}$ indicates a slight distortion of the local octahedron in the equatorial plane resulting
TABLE

The $g$-factors values obtained from the fitting procedure described in the text.

\begin{tabular}{c|c|c|c|c|c|c}
\hline \hline \multirow{2}{*}{} & \multicolumn{2}{|c|}{ Plane $b^{\prime} c^{\prime}$} & \multicolumn{2}{c|}{ Plane $a^{\prime} c^{\prime}$} & \multicolumn{2}{c}{ Plane $a^{\prime} b^{\prime}$} \\
\cline { 2 - 7 } & $4 \mathrm{~K}$ & $300 \mathrm{~K}$ & $4 \mathrm{~K}$ & $300 \mathrm{~K}$ & $4 \mathrm{~K}$ & $300 \mathrm{~K}$ \\
\hline$g_{x}$ & 2.056 & 2.064 & 2.056 & 2.064 & - & - \\
$g_{y}$ & 2.070 & 2.067 & - & - & 2.068 & 2.070 \\
$g_{z}$ & - & - & 2.280 & 2.279 & 2.283 & 2.280 \\
$\alpha$ & $-3.1^{\circ}$ & $5.9^{\circ}$ & $9.2^{\circ}$ & $9.6^{\circ}$ & $10.1^{\circ}$ & $12.0^{\circ}$
\end{tabular}

from the effect of hydrogen bonding which becomes important at low temperatures [7].

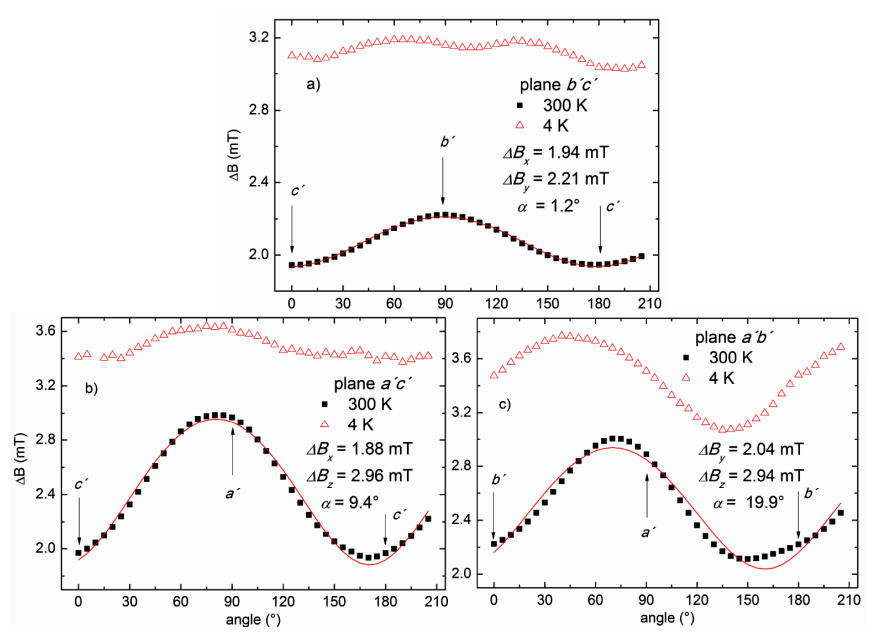

Fig. 3. Angular dependence of EPR linewidth of $\mathrm{Cu}(\mathrm{en})\left(\mathrm{H}_{2} \mathrm{O}\right)_{2} \mathrm{SO}_{4}$. The lines represent the fit described in the text.

In the next step, the angular dependence of the EPR linewidth was analyzed (Fig. 3). The increase of the linewidth observed at low temperatures can be ascribed to the onset of short-range magnetic correlations previously observed in specific-heat and susceptibility studies [4]. Furthermore, the shape of the angular dependence observed at $300 \mathrm{~K}$ qualitatively differs from that observed at $4 \mathrm{~K}$. At room temperature, the angular dependence of the linewidth coincides with the angular dependence of the $g$-factor and can be described by the formula [8]

$$
\begin{aligned}
& \Delta B= \\
& \sqrt{\Delta B_{z}^{2} \cos ^{2} \theta+\Delta B_{x}^{2} \sin ^{2} \theta \cos ^{2} \varphi+\Delta B_{y}^{2} \sin ^{2} \theta \sin ^{2} \varphi} .
\end{aligned}
$$

The deviations between the data and the fitting curve (Fig. 3c) can be ascribed to the monoclinic angle in the $a^{\prime} c^{\prime}$ plane, as was already indicated in the fitting of the $g$-factor (Fig. 2c). Radical changes of the angular dependence of the linewidth appearing at helium temperature can be ascribed to the dipolar coupling, which was not indicated at $300 \mathrm{~K}$ due to the much stronger line broadening originating in the symmetric and antisymmetric exchange coupling, expected in $\mathrm{Cu}(\mathrm{en})\left(\mathrm{H}_{2} \mathrm{O}\right)_{2} \mathrm{SO}_{4}$. The presence of the exchange anisotropy is apparent from the 
$g$-factor anisotropy; the lack of an inversion centre between the $\mathrm{Cu}(\mathrm{II})$ ions in the crystallographic $a b$ plane gives rise to the antisymmetric exchange interaction.

\section{Conclusions}

The angular dependence of EPR spectra of an $\mathrm{Cu}(\mathrm{en})\left(\mathrm{H}_{2} \mathrm{O}\right)_{2} \mathrm{SO}_{4}$ single crystal was investigated at 4 and $300 \mathrm{~K}$. Analysis of the linewidth performed at $300 \mathrm{~K}$ revealed the same angular variation as the $g$-factor. This coincidence is the manifestation of the symmetric and antisymmetric exchange coupling, as the main EPR line-broadening mechanisms in $\mathrm{Cu}(\mathrm{en})\left(\mathrm{H}_{2} \mathrm{O}\right)_{2} \mathrm{SO}_{4}$ at high temperatures originating from the spin-orbit coupling. The change of the angular dependence of the linewidth observed at $4 \mathrm{~K}$ can be ascribed to the dipolar coupling. In the future, more detailed analysis will be performed to estimate the strength of the individual mechanisms responsible for the linewidth broadening.

\section{Acknowledgments}

This work was supported by the project APVV LPP-0202-09, VEGA grant No. 1/0078/09, Deutsche Forschungsgemeinschaft, EuroMagNET (EU contract No. 228043), and ERDF EU project. No.
ITMS26220120005. Material support from U.S. Steel Košice s.r.o. is greatly acknowledged.

\section{References}

[1] V. Schollwöck, J. Richter, D.J.J. Farnell, R.F. Bishop, Quantum Magnetism, Springer-Verlag, Berlin 2004.

[2] K.L. Nagy, B. Náfrádi, N.D. Kushch, E.B. Yagubskii, E. Herdtweck, T. Fehér, L.F. Kiss, L. Forró, A. Jánossy, Phys. Rev. B 80, 104407 (2009).

[3] E. Čižmár, S.A. Zvyagin, R. Beyer, M. Uhlarz, M. Ozerov, Y. Skourski, J.L. Manson, J.A. Schlueter, J. Wosnitza, Phys. Rev. B 81, 064422 (2010).

[4] M. Kajňaková, M. Orendáč, A. Orendáčová, A. Vlček, J. Černák, O.V. Kravchyna, A.G. Anders, M. Bałanda, J.-H. Park, A. Feher, M.W. Meisel, Phys. Rev. B 71, 014435 (2005).

[5] V. Manríquez, M. Campos-Vallette, N. Lara, N. Gonzalez-Tejeda, O. Wittke, G. Diaz, S. Diez, R. Munoz, L. Kriskovic, J. Chem. Crystall. 26, 15 (1996).

[6] S. Mat́áš, private communication.

[7] C. Desplanches, E. Ruiz, A. Rodrıguez-Fortea, S. Alvarez, J. Am. Chem. Soc. 124, 5197 (2002).

[8] M. Dumm, A. Loidl, Phys. Rev. B 62, 6512 (2000). 